\title{
The performance of the EQ-5D-3L in screening for anxiety and depressive symptoms in hospital and community settings
}

\author{
Hilary Short ${ }^{1}$, Fatima Al Sayah ${ }^{1}$, Arto Ohinmaa ${ }^{2}$ and Jeffrey A. Johnson ${ }^{{ }^{*}}$ (D)
}

\begin{abstract}
Background: To examine the performance of the EQ-5D-3L in screening for anxiety and depressive symptoms in hospital and community settings compared to other patient-reported screening tools.

Methods: Data from a prospective cohort of patients discharged from general internal medicine wards from two hospitals in Edmonton, Alberta were used in this study. Two waves of measurements (discharge and 90-days post-discharge) were analyzed. The performance of the EQ-5D-3L was compared to other self-report screening tools: Generalized Anxiety Disorder 2-item questionnaire was used to categorize anxiety symptoms into absent $(<3)$ and present $(\geq 3)$, and the Patient Health Questionnaire 9-items was used to categorize depressive symptoms by two severity cut-points: no $(<10)$ vs. mild $(\geq 10)$, and no $(<15)$ vs. moderate-severe $(\geq 15)$. Performance of EQ-5D-3L in screening for anxiety and depressive symptoms was evaluated using receiver operating curve (ROC) analysis.
\end{abstract}

Results: Average age of participants ( $n=493$ ) was 62.9 years (SD 18.6); $51 \%$ were female. At discharge, $30.0 \%$ screened positive for mild and $12.8 \%$ for moderate-severe depressive symptoms, while $27.6 \%$ screened positive for anxiety symptoms. For co-morbid symptoms, $17.1 \%$ screened positive for anxiety and any depressive symptoms, while 10.8\% for anxiety and moderate-severe depressive symptoms. While the EQ-5D-3L had limited screening ability in hospital, the anxiety/depression dimension performed well in the community setting (90-days post-discharge) in screening for anxiety (area under ROC 0.79), depressive symptoms (any: 0.78, moderate-severe: 0.84 ), and a combination of both (any: 0.86; moderate-severe: 0.91).

Conclusions: The EQ-5D-3L anxiety/depression dimension could be a useful tool in screening for anxiety and depressive symptoms in community settings compared to other self-report screening tools. The usefulness of the EQ5D-3L as a screening tool in other settings and populations is warranted.

Keywords: Screening, Depression, Anxiety, EQ-5D-3L, Hospital, Community setting

\section{Background}

Physical and mental health have a complex, bidirectional relationship [1]. Poor mental health is associated with increased risk of physical illness and poor physical health is related to higher risk for mental disorders, most

\footnotetext{
*Correspondence: jeff.johnson@ualberta.ca

1 2-040 Li Ka Shing Centre for Health Research Innovation, School of Public Health, University of Alberta, Edmonton, AB T6G 2E1, Canada

Full list of author information is available at the end of the article
}

commonly depression and anxiety [1]. Mental disorders are common and rank as the leading cause of disability in Canada [2]. The chance of having a mental illness in a Canadian lifetime is 1 in 5 [2]. Estimated expenditure on non-dementia-related mental healthcare in Canada was $\$ 15.8$ billion in 2015 [3], yet one-third of adults may not have their mental health care needs fully met [4]. Healthcare systems should be strengthened to improve the delivery on mental health care, particularly in 'first point of contact' settings such as hospitals and primary care. original author(s) and the source, provide a link to the Creative Commons licence, and indicate if changes were made. The images or other third party material in this article are included in the article's Creative Commons licence, unless indicated otherwise in a credit line to the material. If material is not included in the article's Creative Commons licence and your intended use is not permitted by statutory regulation or exceeds the permitted use, you will need to obtain permission directly from the copyright holder. To view a copy of this licence, visit http://creativecommons.org/licenses/by/4.0/. The Creative Commons Public Domain Dedication waiver (http://creativeco mmons.org/publicdomain/zero/1.0/) applies to the data made available in this article, unless otherwise stated in a credit line to the data. 
Significant mental disorders occur in about $30 \%$ of hospitalized inpatients [5], however, less than $5 \%$ of admissions receive a mental health assessment [6-8]. Inpatient care often focuses on treating patients' presenting illness or injury and discharging them as quickly as possible [9]. Referrals to psychiatric consultation or liaison services are low [10], and inadequate identification and management of mental disorders has significant implications [11]. Hospitalized patients with undiagnosed, and therefore untreated, mental disorders have poorer outcomes $[9,12,13]$, increased mortality [14], extended hospitalization $[9,12,13,15]$, greater risk for hospital readmission [11-13], greater use of medical services [16, 17], and reduced compliance with treatment [9].

It has been estimated that $80 \%$ of people with major depressive disorder are treated entirely in the primary care setting, resulting in depression being one of the most prevalent and fastest rising disorders in primary care [18]. Additionally, generalized anxiety disorder is the most frequent anxiety disorder in primary care, with $6 \%$ to $22 \%$ of primary care patients complaining of anxiety problems, which suggests that these patients are high users of primary care resources [19-21]. However, mental disorders are frequently under-diagnosed in primary care [22]. Studies show that primary care physicians recognize depressive episodes less than $50 \%$ of the time [23] and identify anxiety and mood disorders at "chance levels of probability" [24, 25]. Many individuals may suffer from mental disorders for years before receiving appropriate treatment, resulting in significant morbidity for those who suffer from these disorders in addition to social and economic costs [18].

Hospitalization and primary care visits represent unrecognized opportunities to improve both mental and physical health outcomes [9]. Evidence shows that common mental disorders can be effectively treated in people with a physical health condition [1], and integrated mental healthcare positively impacts physical health outcomes [26] and reduces services use and healthcare costs $[27,28]$. However, diagnosing mental disorders in the physically unwell can be challenging for the examining physician [29]. Furthermore, hospital and primary care physicians may view mental disorders as out of their scope of practice and a problem to be managed by other health care professionals [11].

Given the high rate of under-diagnosed mental disorders, a suitable screening instrument for the inpatient and outpatient environment to aid the diagnostic process would be useful. While screening measures do not replace a clinical diagnosis, an effective screening measure can prompt health care providers to further assess patients' mental health and refer patients that may have been overlooked to a mental health professional.
In Canadian healthcare settings, there is no consistent approach of screening for mental health symptoms. Patient-reported outcome measures (PROMs) of general health status have been increasingly used for routine outcome measurements in health systems in Canada and internationally [9]. The EQ-5D, which includes an item assessing anxiety and depression, is a commonly used generic measure in routine outcome measurement initiatives. It has been suggested that a single question on depressed mood can detect $85-90 \%$ of patients with major depressive disorder [30]. More elaborate screening tools have not attained broad acceptance by general physicians due to the time involved in administering and interpreting them, as well as their focus on the diagnosis and detailed diagnostic criteria of mental illness. The Diagnostic and Statistical Manual of Mental Disorders, Fourth Edition (DSM-IV) is of limited utility for primary care or physicians or internists $[25,31]$ who may not have the time or inclination to determine whether a patients' emotional distress might meet criteria for a mental illness. In fact, it's been shown that half of primary care physicians often do not use the DSM-IV diagnostic criteria when diagnosing depression [32].

Within Alberta, Canada, the healthcare system endorsed and embedded the EQ-5D in Connect Care, the province-wide hospital-based electronic patient medical information system, and is widely collected across the healthcare system. Additionally, the EQ-5D has been adopted for clinical use within Alberta's Primary Care Networks. This tool offers an opportunity for a standardized approach to routine mental health screening if it is found fit for this purpose [33]. Given the crucial need for improved anxiety and depression detection, we sought to examine whether the EQ-5D might be suitable as a mental health screening tool, compared to other self-report screening tools, in the hospital setting as well as after discharge in the community setting.

\section{Methods \\ Data source}

This study was a secondary analysis of data from a prospective cohort of patients 18 years of age and older who were being discharged from seven general internal medicine wards from two hospitals in Edmonton (University of Alberta and Royal Alexandra hospitals) between October 2013 and November 2014 [34]. Patients were excluded if they did not live in the province, had severe cognitive impairment, were deemed by their attending physician to have fore-shortened life expectancy that would preclude 90-day follow up, or were transferred to or from a long-term care facility, another inpatient service or another acute care hospital [34]. Data collected at the time of discharge was used to evaluate the EQ-5D-3L 
screening performance in the hospital setting, and data from 90-days post discharge, collected by telephone interview, was used for the community setting.

\section{Measures}

The EQ-5D-3L is a generic preference-based measure of health-related quality of life. It measures five dimensions of health (mobility, self-care, usual activities, pain/ discomfort, and anxiety/depression), by three levels of perceived problems: no problems "1", some/moderate problems "2", extreme problems/unable to/confined to bed " 3 " [35]. The EQ-5D-3L describes 243 unique health states defined by combining one level from each of the five dimensions; 11111 is the best health state (no problems on any of the dimensions) and 33333 is the worst health state (extreme problems on all of the dimensions) [35]. The reference period of the EQ-5D is "today". The EQ-5D-3L also includes a visual analogue scale (EQVAS), which represents the respondent's self-rated health 'today' on a scale ranging from 0 "worst imaginable health state" to 100 "best imaginable health state" [35]. Population value sets are used to derive index scores for each health state [36]. We used the EQ-5D-3L Canadian value set to calculate index scores in this study [37].

The performance of the EQ-5D-3L (anxiety/depression dimension and index score) in screening for anxiety and depressive symptoms was evaluated in comparison to two self-report screening instruments: The Generalized Anxiety Disorder 2-item (GAD-2) questionnaire and the Patient Health Questionnaire 9-items (PHQ-9).

GAD-2 assesses the presence and frequency of anxiety symptoms "over the last 2 weeks" [38]. It includes two items that are scored from 0 (not at all) to 3 (nearly every day), with higher scores indicating higher severity of anxiety symptoms. A total score was generated by summing the two items (range 0-6) and presence of anxiety symptoms is indicated by a cut-off point of $\geq 3$ [38]. A score of 3 points is the preferred cut-off for identifying possible cases, and in which further assessment for generalized anxiety disorder is warranted [39]. The total score was categorized as present $(\mathrm{GAD}-2 \geq 3)$ vs. absent $($ GAD $-2<3)$ anxiety symptoms. The sensitivity and specificity of the GAD-2 in identifying generalized anxiety disorder in primary care were reported to be $86 \%$ and $83 \%$, respectively [38]. Despite the GAD questionnaire being a commonly used screening instruments for anxiety symptoms, there are no studies reporting its usefulness in hospital or inpatient settings.

The PHQ-9 measures the presence and frequency of depressive symptoms "over the last 2 weeks". All 9 items are scored from 0 (not at all) to 3 (nearly every day), with higher scores indicating higher severity of depressive symptoms. A total score is generated by summing the scores of the 9 items (range 0-27) [40]. A meta-analysis on the use of the PHQ-9 in screening for major depressive disorder in primary care and obstetrics-gynecology patients indicated that the pooled sensitivity and specificity estimates of the PHQ- 9 were $78 \%$ and $87 \%$, respectively [39]. A validation study of the PHQ-9 in assessing major depressive disorder during inpatient spinal cord rehabilitation reported $100 \%$ sensitivity and $84 \%$ specificity [41]. The total PHQ-9 score was categorized into two severity levels:

- Any depressive symptoms (PHQ-9 $\geq 10$ ) vs. absent depressive symptoms (PHQ-9<10) [40]

- Moderate-severe depressive symptoms (PHQ-9 $\geq 15$ ) vs. absent moderate-severe depressive symptoms PHQ-9<15) [40]

\section{Statistical analysis}

Descriptive statistics were computed for demographic variables in the sample at the time of discharge. Chisquare tests and analysis of variances (ANOVA) were used as appropriate to examine the differences between healthy and anxious and/or depressed groups in mean index scores and distribution of problems in the anxiety/depression dimension. The EQ-5D-3L index score was categorized into tertiles. The anxiety/depression dimension has three levels; $1=$ not anxious or depressed, $2=$ moderately anxious or depressed, $3=$ extremely anxious or depressed and was used as such in the analysis. In addition to examining anxiety and depressive symptoms based on GAD-2 and PHQ-9 as separate variables, we created two composite variables, one for each cut-off point of PHQ-9 (any; moderate-severe), as follows:

- Absent anxiety and depressive symptoms

- Present anxiety and no depressive symptoms

- Absent anxiety and present depressive symptoms

- Present anxiety and depressive symptoms

Area under receiver operating curve (AUROC) analysis was used to examine the performance of the EQ-5D-3L components (anxiety/depression dimension and index score) in screening for anxiety and depressive symptoms. Sensitivity, specificity, positive ( $L R+$ ) and negative ( $L R-$ ) likelihood ratios for presence of anxiety symptoms and the two severity levels of depressive symptoms at each cut-point, as well as an overall AUROC with 95\% confidence interval $(\mathrm{CI})$ were calculated to identify the optimal threshold for each of the examined components. AUROC values were interpreted as follows: $\leq 0.5$ non-informative, $0.5<$ AUROC $\leq 0.7$ less accurate, $0.7<$ AUROC $\leq 0.9$ moderately accurate, $0.9<$ AUROC $<1.0$ highly accurate, 
and $A U R O C=1$ perfect test [42]. All analyses were performed using STATA 14.2 [43].

\section{Results}

\section{General characteristics of participants}

Of 720 eligible patients discharged from the medical wards during the study period, 493 enrolled $(69 \%$ response rate). At 90 -days post-discharge, there was 19\% loss to follow-up $(\mathrm{N}=402)$. Average age of participants at the time of discharge from hospital $(\mathrm{N}=493)$ was 62.9 years (SD 18.6), half were female (51\%), 82\% were White, $49 \%$ were retired, and over half (58\%) were single, widowed or separated. Nearly half (46\%) had more education than a high school diploma, and more than two thirds (68\%) had an annual household income of less than $\$ 80,000 \mathrm{CAD}$. On average participants had 4.9 comorbidities (SD 2.8) and reasons for hospitalization varied significantly (Table 1).

At the time of discharge, the mean GAD-2 score was 1.7 (SD 1.8), and $28 \%$ screened positive for anxiety symptoms (GAD-2 $\geq 3$ ), while the mean PHQ-9 score was 7.4 (SD 5.8), and 30\% screened positive for any depressive symptoms (PHQ-9 $\geq 10$ ), and $12.8 \%$ screened positive for moderate-severe depressive symptoms (PHQ-9 $\geq 15$ ). Further, $17 \%$ screened positive for co-morbid anxiety and any depressive symptoms (GAD- $2 \geq 3$ and PHQ-9 $\geq 10$ ), and $11 \%$ screened positive for co-morbid anxiety and moderate-severe depressive symptoms (GAD-2 $\geq 3$ and PHQ-9 $\geq 15$ ). The mean EQ-5D-3L index score was 0.69 (SD 0.21), with $42 \%$ reporting some to extreme (levels 2-3) problems on the anxiety/ depression dimension (Table 2).

Individuals with anxiety and/or depressive symptoms, based on GAD-2 and PHQ-9 scores, had a considerably lower EQ-5D-3L index and EQ VAS scores compared to those without any symptoms ( 0.75 and 66.9 , respectively): anxiety $(0.63,59.2$, respectively), any depressive symptoms $(0.64,63.7$, respectively), moderate-severe depressive symptoms $(0.64,62.8$, respectively), comorbid anxiety and any depressive symptoms $(0.59,54.6$, respectively), and comorbid anxiety and moderate-severe depressive symptoms $(0.57,50.7$, respectively) (Table 3$)$.

\section{Performance of EQ-5D-3L in screening for anxiety symptoms}

The EQ-5D-3L anxiety/depression dimension was moderately accurate in screening for anxiety symptoms compared to the GAD-2 (Table 4), whereby the highest performance was in the community setting (AUROC $0.79,95 \% \mathrm{CI}: 0.74,0.85)$ with an optimal performance cut-off point at $\geq 2$ (sensitivity $74.6 \%$ ), and the poorest performance in the hospital setting (AUROC 0.75, 95\% CI: $0.70,0.79$ ). The performance of the EQ-5D-3L index
Table 1 Sample characteristics at baseline

\begin{tabular}{|c|c|}
\hline Mean \pm SD or N (\%) & $\begin{array}{l}\text { Baseline/ } \\
\text { discharge } \\
(\mathrm{N}=493)\end{array}$ \\
\hline Age (years) & $62.9 \pm 18.6$ \\
\hline Sex (female) & $250(51 \%)$ \\
\hline \multicolumn{2}{|l|}{ Marital status } \\
\hline Married/common-law & $209(42.2 \%)$ \\
\hline Single/divorced/widowed & $286(57.8 \%)$ \\
\hline \multicolumn{2}{|l|}{ Smoking status } \\
\hline Current & $118(23.8 \%)$ \\
\hline Former & $218(44.0 \%)$ \\
\hline Never & $159(32.1 \%)$ \\
\hline \multicolumn{2}{|l|}{ Education level } \\
\hline$<$ high school & $188(38.0 \%)$ \\
\hline High school diploma & $78(15.8 \%)$ \\
\hline > high school & $229(46.3 \%)$ \\
\hline Employment status (retired) & $243(49.1 \%)$ \\
\hline \multicolumn{2}{|l|}{ Total household income \$CAD } \\
\hline$<80,000$ & $337(68.2 \%)$ \\
\hline$\geq 80,000$ & $90(18.2 \%)$ \\
\hline \multicolumn{2}{|l|}{ Living situation (prior to admission) } \\
\hline Home without any support & $289(53.4 \%)$ \\
\hline Home, informal caregiver & $90(18.2 \%)$ \\
\hline Home, with homecare & $76(15.4 \%)$ \\
\hline Home, with live-in caregiver & $3(0.6 \%)$ \\
\hline Assisted living/lodge & $37(7.5 \%)$ \\
\hline \multicolumn{2}{|l|}{ Ethnicity } \\
\hline White & $407(82.2 \%)$ \\
\hline Other & $81(16.3 \%)$ \\
\hline \multicolumn{2}{|l|}{ Reason for hospitalization } \\
\hline Heart failure & $50(10.1 \%)$ \\
\hline Pneumonia/empyema/lung abscess & $49(9.9 \%)$ \\
\hline Diabetes & $26(5.3 \%)$ \\
\hline Urinary tract infection & $26(5.3 \%)$ \\
\hline Venous thromboembolic disease (PE/DVT) & $26(5.3 \%)$ \\
\hline Cellulitis/decubitus ulcers & $16(3.2 \%)$ \\
\hline Sepsis & $12(2.4 \%)$ \\
\hline $\mathrm{ETOH}$ or street drug intoxication/withdrawal & $12(2.4 \%)$ \\
\hline Gastroenteritis/colitis & $12(2.4 \%)$ \\
\hline Cancer & $11(2.2 \%)$ \\
\hline Acute kidney injury & $11(2.2 \%)$ \\
\hline Pancreatitis & $10(2.0 \%)$ \\
\hline Other & $234(52.7 \%)$ \\
\hline Number of comorbidities & $4.9 \pm 2.8$ \\
\hline
\end{tabular}

and EQ VAS scores in screening for anxiety were poor with respective AUROC of 0.37 (95\% CI: $0.32,0.42$ ) and 0.40 (95\% CI: $0.33,0.47)$ in hospital, and 0.27 (95\% CI $0.22,0.33)$ and 0.33 (95\% CI: $0.27,0.39)$ in community setting. 
Table 2 Anxiety, depression, and health status

\begin{tabular}{|c|c|c|}
\hline Mean \pm SD or N (\%) & $\begin{array}{l}\text { Hospital } \\
\text { Setting } \\
(N=493)\end{array}$ & $\begin{array}{l}\text { Community } \\
\text { Setting } \\
(N=402)\end{array}$ \\
\hline \multicolumn{3}{|l|}{$E Q-5 D-3 L$} \\
\hline Anxiety/depression dimension (levels 2-3) & $209(42.2 \%)$ & $108(26.9 \%)$ \\
\hline Index score & $0.69 \pm 0.21$ & $0.77 \pm 0.22$ \\
\hline VAS & $63.4 \pm 18.9$ & $71.4 \pm 19.4$ \\
\hline \multicolumn{3}{|l|}{ PHQ-9 } \\
\hline Total score & $7.4 \pm 5.8$ & $4.0 \pm 4.6$ \\
\hline$\geq 10$ & $148(30.0 \%)$ & $49(12.2 \%)$ \\
\hline$\geq 15$ & $63(12.8 \%)$ & $14(3.5 \%)$ \\
\hline \multicolumn{3}{|l|}{ GAD-2 } \\
\hline Total score & $1.7 \pm 1.8$ & $1.0 \pm 1.6$ \\
\hline$\geq 3$ & $136(27.6 \%)$ & $67(16.7 \%)$ \\
\hline \multicolumn{3}{|l|}{ Composite variable $1^{a}$} \\
\hline - anxiety - depression & $292(59.4 \%)$ & $310(77.5 \%)$ \\
\hline + anxiety - depression & $64(13.0 \%)$ & $23(5.8 \%)$ \\
\hline - anxiety + depression & $52(10.6 \%)$ & $41(10.3 \%)$ \\
\hline+ anxiety + depression & $84(17.1 \%)$ & $26(6.5 \%)$ \\
\hline \multicolumn{3}{|l|}{ Composite variable $2^{b}$} \\
\hline - anxiety - moderate-severe depression & $346(70.3 \%)$ & $329(82.3 \%)$ \\
\hline + anxiety - moderate-severe depression & $10(2.0 \%)$ & $4(1.0 \%)$ \\
\hline - anxiety + moderate-severe depression & $83(16.9 \%)$ & $57(14.3 \%)$ \\
\hline+ anxiety + moderate-severe depression & $53(10.8 \%)$ & $10(2.5 \%)$ \\
\hline
\end{tabular}

\section{Performance of EQ-5D-3L in screening for depressive symptoms}

The EQ-5D-3L anxiety/depression dimension performance in screening for depressive symptoms compared to the PHQ-9 was moderate (Table 5). For any depressive symptoms (PHQ-9 $\geq 10$ ), the highest AUROC was in the community setting $(0.78,95 \% \mathrm{CI}: 0.71,0.85)$ with an optimal cut-off point $\geq 2$ (sensitivity $73.5 \%$ ). For moderate-severe depression (PHQ-9 $\geq 15$ ), the highest AUROC was also in the community setting (0.84, 95\% CI: $0.73,0.94$ ) with an optimal cut-off point $\geq 2$ (sensitivity $85.7 \%$ ). The EQ-5D-3L anxiety/depression dimension performed better in screening for moderate-severe depressive symptoms than for mild symptoms. The performance of this dimension in screening for depressive symptoms in the hospital setting was poor. Additionally, the performance of the EQ-5D-3L index and EQ VAS scores in screening for depressive symptoms were also poor in both hospital and community settings.
Performance of EQ-5D-3L in screening for comorbid anxiety and depressive symptoms

The EQ-5D-3L anxiety/depression dimension performed better in screening for comorbid anxiety and depressive symptoms compared to its performance in screening for each of these symptoms alone. For comorbid anxiety and any depressive symptoms, the anxiety/depression dimension AUROC was 0.86 (95\% CI: 0.80, 0.92) in the community setting with an optimal cut-off point $\geq 2$ (sensitivity $92.3 \%$ ) compared to 0.74 (95\% CI $0.68,0.79)$ in hospital setting (Table 6). For comorbid anxiety and moderatesevere depression, the anxiety/depression dimension AUROC was 0.91 (95\% CI: 0.87, 0.95) in the community setting with an optimal cut-off point $\geq 2$ (sensitivity $100.0 \%)$, compared to 0.73 (95\% CI 0.67, 0.79) in hospital setting. The performance of the EQ-5D-3L index and EQ VAS scores in screening for comorbid anxiety and depressive symptoms were poor in both hospital and community settings and for both levels of depressive symptoms.

\section{Discussion}

In this study, we found that the EQ-5D-3L anxiety/ depression dimension performed well in screening for anxiety and depressive symptoms in adults in the community setting; it was less useful in the hospital setting, particularly at the time of discharge. Additionally, the screening performance of this dimension was better when anxiety and depressive symptoms were both present than either symptom alone. Further, this dimension was more accurate for "moderate-severe" levels of depressive symptoms, either alone or with anxiety, than for "any" levels of depressive symptoms. The screening performance of the EQ-5D-5L anxiety/depression dimension was similar to previously validated, but separate, screening measures for each of the set of symptoms. Finally, we found that the EQ-5D-3L index and EQ VAS scores generally had poor performance in screening for anxiety and/or any level of depressive symptoms in both hospital and community settings.

The proportion of patients reporting problems on the anxiety/depression dimension (levels 2-3) in hospital and community settings were $42 \%$ and $27 \%$, respectively; higher than the Alberta population norm, 23\% [44]. Lower EQ-5D-3L index and EQ VAS scores were observed in patients with anxiety and depressive symptoms and more considerably among the co-morbid anxiety and depressive symptoms groups, with the lowest index and EQ VAS scores observed at 0.45 and 49.0, respectively. These overall scores were nonetheless also picking up the considerable physical health problems in this sample. It is evident that there is an emotional and 


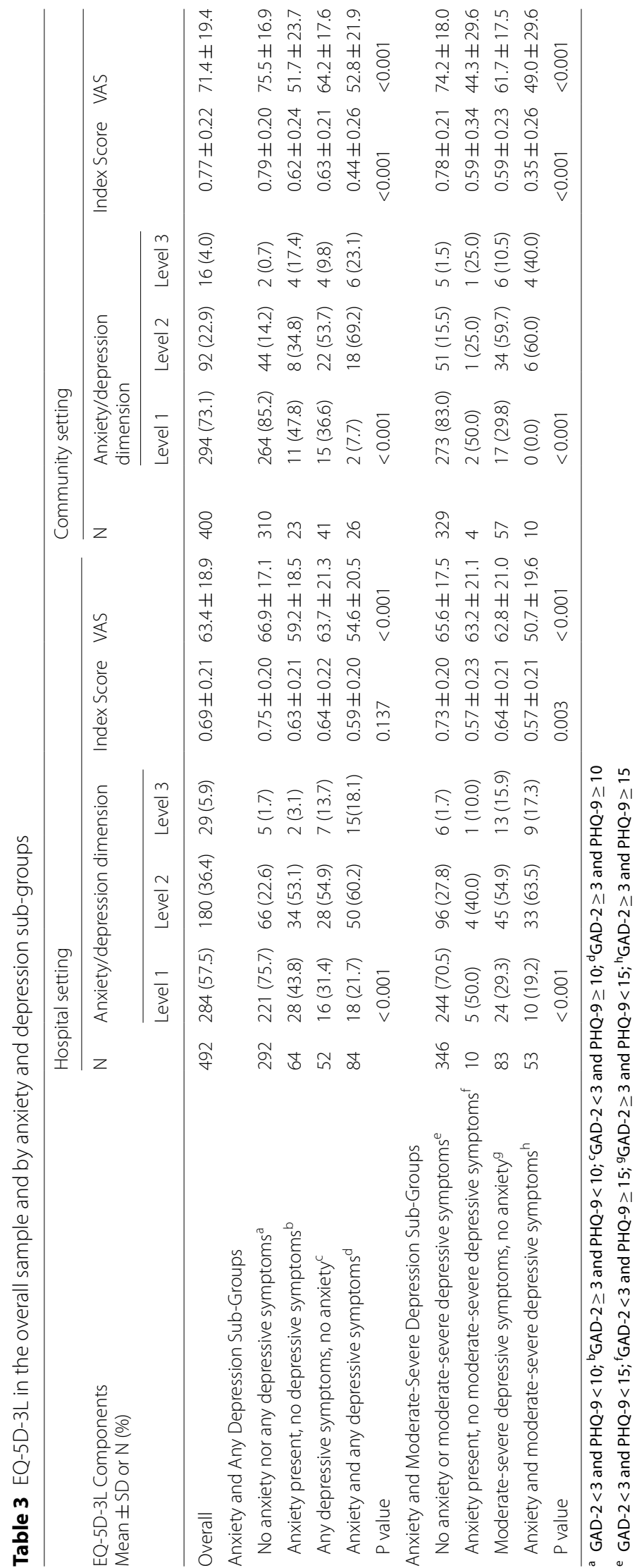


Table 4 Performance of the EQ-5D-3L in screening for anxiety symptoms (GAD-2 $\geq 3$ )

\begin{tabular}{|c|c|c|c|c|}
\hline \multirow[t]{2}{*}{ Cut-point } & \multicolumn{2}{|c|}{ Hospital setting $(N=492)$} & \multicolumn{2}{|c|}{ Community setting $(\mathrm{N}=400)$} \\
\hline & $\geq 2$ & $\geq 3$ & $\geq 2$ & $\geq 3$ \\
\hline \multicolumn{5}{|c|}{ EQ-5D-3L Anxiety/depression dimension } \\
\hline Sensitivity & $75.0 \%$ & $17.7 \%$ & $74.6 \%$ & $14.9 \%$ \\
\hline Specificity & $69.8 \%$ & $98.0 \%$ & $82.6 \%$ & $98.2 \%$ \\
\hline $\mathrm{LR}+$ & 2.48 & 9.00 & 4.28 & 8.28 \\
\hline LR- & 0.36 & 0.84 & 0.31 & 0.87 \\
\hline PPV & $48.0 \%$ & $76.6 \%$ & $45.0 \%$ & $61.2 \%$ \\
\hline NPV & $88.3 \%$ & $76.3 \%$ & $94.5 \%$ & $85.8 \%$ \\
\hline AUROC (95\% Cl) & $0.74(0.70,0.79)$ & & $0.79(0.74,0.85)$ & \\
\hline \multicolumn{5}{|c|}{ EQ-5D-3L index score tertiles } \\
\hline Sensitivity & $52.2 \%$ & $14.7 \%$ & $25.4 \%$ & $4.5 \%$ \\
\hline Specificity & $30.3 \%$ & $62.8 \%$ & $34.4 \%$ & $68.3 \%$ \\
\hline LR+ & 0.75 & 0.39 & 0.39 & 0.14 \\
\hline LR- & 1.58 & 1.36 & 2.17 & 1.40 \\
\hline PPV & $21.7 \%$ & $12.8 \%$ & $6.9 \%$ & $2.6 \%$ \\
\hline NPV & $63.2 \%$ & $66.6 \%$ & $70.8 \%$ & $79.0 \%$ \\
\hline AUROC (95\% Cl) & $0.37(0.32,0.42)$ & & $0.27(0.22,0.33)$ & \\
\hline \multicolumn{5}{|c|}{ EQ-VAS score tertiles } \\
\hline Sensitivity & $52.9 \%$ & $13.2 \%$ & $41.8 \%$ & $10.5 \%$ \\
\hline Specificity & $32.4 \%$ & $73.0 \%$ & $30.5 \%$ & $68.0 \%$ \\
\hline LR+ & 0.78 & 0.49 & 0.60 & 0.33 \\
\hline LR- & 1.45 & 1.19 & 1.91 & 1.32 \\
\hline PPV & $22.5 \%$ & $15.3 \%$ & $10.3 \%$ & $5.9 \%$ \\
\hline NPV & $65.0 \%$ & $69.5 \%$ & $73.3 \%$ & $80.0 \%$ \\
\hline AUROC (95\% Cl) & $0.40(0.33,0.47)$ & & $0.33(0.27,0.39)$ & \\
\hline
\end{tabular}

$\mathrm{LR}+$, positive likelihood ratio; $\mathrm{LR}-$, negative likelihood ratio; $A U R O C$, area under receiver operating curve. EQ-5D-3L index score tertiles: $2=$ quintile $2,3=$ quintile 3. EQ-5D-3L anxiety/depression dimension: 2 = moderately anxious or depressed, $3=$ extremely anxious or depressed. PPV, positive predictive value; NPV, negative predictive value. Bolded values indicate the cut-point that maximizes sensitivity and specificity for each component

overall health-related quality of life burden associated with anxiety and/or depressive symptoms, especially when compared to the overall Alberta adult population norms of the EQ-5D-3L index and EQ VAS scores of 0.93 and 78.3, respectively [44].

Several studies have supported the use of the EQ5D-3L for measuring health status in individuals with mental health problems [14-16], but only one other study has shown that the EQ-5D-3L is a useful screening tool for anxiety and depressive symptoms in the general population [17]. Supina et al. (2007) examined the ability of the EQ-5D-3L in differentiating those with a clinical diagnosis of major depressive disorder and/ or an anxiety disorder. The authors found that major depression disorder is slightly more predictive of problems reported (levels 2-3) on the anxiety/depression dimension than anxiety alone, and the co-morbidity of depression and anxiety resulted in a significantly greater likelihood of respondents reporting problems. Additionally, Al Sayah et al. (2018) investigated the performance of the EQ-5D-5L in screening for anxiety and depressive symptoms in type 2 diabetes patients in an outpatient community setting. They found that the EQ-5D-5L anxiety/depression dimension performed very well in screening for anxiety (AUROC: 0.89), depressive symptoms (any: 0.88, moderate-severe: 0.90 ), and comorbid anxiety and depressive symptoms (any: 0.92, moderate-severe: 0.92) [10]. These two studies provide similar evidence to our study and support our findings that the EQ-5D-3L is more appropriate in a community setting. Preference based measures, like the EQ-5D-3L, are commonly used for economic evaluations, but are increasingly being used in community health care settings for quality improvement purposes, patient management, and surveillance of population health [10]. Routine mental health screening using a generic PROM, like the EQ-5D-3L, could also be implemented in addition to these large-scale community health applications [10].

Our findings suggest that the EQ-5D-3L, specifically the anxiety/depression dimension, would be more suitable in screening for anxiety and/or depressive symptoms in an outpatient community setting rather than the 
Table 5 Performance of the EQ-5D-3L in screening for depressive symptoms

\begin{tabular}{|c|c|c|c|c|}
\hline \multirow[t]{2}{*}{ Cut-point } & \multicolumn{2}{|c|}{ Hospital setting $(\mathrm{N}=492)$} & \multicolumn{2}{|c|}{ Community setting $(\mathrm{N}=400)$} \\
\hline & $\geq 2$ & $\geq 3$ & $\geq 2$ & $\geq 3$ \\
\hline \multicolumn{5}{|c|}{ Any depressive symptoms (PHQ-9 $\geq 10$ ) } \\
\hline \multicolumn{5}{|c|}{ EQ-5D-3L Anxiety/depression dimension } \\
\hline Sensitivity & $68.9 \%$ & $12.2 \%$ & $73.5 \%$ & $20.4 \%$ \\
\hline Specificity & $68.8 \%$ & $96.2 \%$ & $79.6 \%$ & $98.3 \%$ \\
\hline LR+ & 2.21 & 3.24 & 3.59 & 11.97 \\
\hline LR- & 0.45 & 0.91 & 0.33 & 0.81 \\
\hline PPV & $48.5 \%$ & $57.8 \%$ & $33.4 \%$ & $62.5 \%$ \\
\hline NPV & $83.8 \%$ & $72.0 \%$ & $95.6 \%$ & $89.9 \%$ \\
\hline AUROC $(95 \% \mathrm{Cl})$ & $0.70(0.65,0.74)$ & & $0.78(0.71,0.85)$ & \\
\hline \multicolumn{5}{|c|}{ EQ-5D-3L index score tertiles } \\
\hline Sensitivity & $48.7 \%$ & $13.5 \%$ & $28.6 \%$ & $4.1 \%$ \\
\hline Specificity & $28.0 \%$ & $61.6 \%$ & $36.7 \%$ & $69.6 \%$ \\
\hline LR+ & 0.68 & 0.35 & 0.45 & 0.13 \\
\hline$L R-$ & 1.83 & 1.40 & 1.95 & 1.38 \\
\hline PPV & $22.4 \%$ & $13.0 \%$ & $5.9 \%$ & $1.8 \%$ \\
\hline NPV & $56.1 \%$ & $62.5 \%$ & $78.7 \%$ & $83.9 \%$ \\
\hline AUROC $(95 \% \mathrm{Cl})$ & $0.34(0.29,0.39)$ & & $0.30(0.24,0.36)$ & \\
\hline \multicolumn{5}{|c|}{ EQ-VAS score tertiles } \\
\hline Sensitivity & $51.0 \%$ & $14.3 \%$ & $28.5 \%$ & $6.1 \%$ \\
\hline Specificity & $29.0 \%$ & $71.0 \%$ & $29.8 \%$ & $68.5 \%$ \\
\hline LR+ & 0.72 & 0.49 & 0.41 & 0.19 \\
\hline LR- & 1.69 & 1.21 & 2.40 & 1.37 \\
\hline PPV & $23.5 \%$ & $17.4 \%$ & $5.3 \%$ & $2.6 \%$ \\
\hline NPV & $58.1 \%$ & $66.0 \%$ & $75.0 \%$ & $84.0 \%$ \\
\hline AUROC $(95 \% \mathrm{Cl})$ & $0.38(0.33,0.43)$ & & $0.27(0.20,0.33)$ & \\
\hline \multicolumn{5}{|c|}{ Moderate-severe symptoms (PHQ-9 $\geq 15)$} \\
\hline \multicolumn{5}{|c|}{ EQ-5D-3L Anxiety/depression dimension } \\
\hline Sensitivity & $76.2 \%$ & $17.5 \%$ & $85.7 \%$ & $35.7 \%$ \\
\hline Specificity & $62.4 \%$ & $95.4 \%$ & $75.2 \%$ & $97.2 \%$ \\
\hline LR+ & 2.03 & 3.76 & 3.46 & 12.56 \\
\hline LR- & 0.38 & 0.87 & 0.19 & 0.66 \\
\hline PPV & $22.6 \%$ & $35.4 \%$ & $11.4 \%$ & $31.6 \%$ \\
\hline NPV & $94.8 \%$ & $88.9 \%$ & $99.3 \%$ & $97.7 \%$ \\
\hline AUROC $(95 \% \mathrm{Cl})$ & $0.71(0.65,0.77)$ & & $0.84(0.73,0.94)$ & \\
\hline \multicolumn{5}{|c|}{ EQ-5D-3L index score tertiles } \\
\hline Sensitivity & $44.4 \%$ & $11.1 \%$ & $21.4 \%$ & $0.0 \%$ \\
\hline Specificity & $32.0 \%$ & $66.1 \%$ & $39.5 \%$ & $71.8 \%$ \\
\hline LR+ & 0.65 & 0.33 & 0.35 & 0.00 \\
\hline$L R-$ & 1.74 & 1.34 & 1.99 & 1.39 \\
\hline PPV & $8.6 \%$ & $4.5 \%$ & $1.3 \%$ & $0.0 \%$ \\
\hline NPV & $80.0 \%$ & $83.8 \%$ & $93.3 \%$ & $95.2 \%$ \\
\hline AUROC $(95 \% \mathrm{Cl})$ & $0.34(0.28,0.41)$ & & $0.27(0.19,0.36)$ & \\
\hline \multicolumn{5}{|l|}{ EQ-VAS score tertiles } \\
\hline Sensitivity & $45.2 \%$ & $8.1 \%$ & $28.6 \%$ & $7.1 \%$ \\
\hline Specificity & $32.1 \%$ & $73.0 \%$ & $33.6 \%$ & $70.8 \%$ \\
\hline $\mathrm{LR}+$ & 0.67 & 0.30 & 0.43 & 0.24 \\
\hline$L R-$ & 1.71 & 1.26 & 2.13 & 1.31 \\
\hline PPV & $8.8 \%$ & $4.2 \%$ & $1.5 \%$ & $0.9 \%$ \\
\hline NPV & $80.3 \%$ & $84.6 \%$ & $92.8 \%$ & $95.5 \%$ \\
\hline AUROC $(95 \% \mathrm{Cl})$ & $0.35(0.29,0.42)$ & & $0.29(0.17,0.41)$ & \\
\hline
\end{tabular}


Table 5 (continued)

$\mathrm{LR}+$, positive likelihood ratio; $\mathrm{LR}-$, negative likelihood ratio; $\mathrm{AUROC}$, area under receiver operating curve; EQ-5D-3L index score tertiles: $2=$ quintile $2,3=$ quintile 3. EQ-5D-3L anxiety/depression dimension: 2 = moderately anxious or depressed, $3=$ extremely anxious or depressed. PPV, positive predictive value; NPV, negative predictive value. Bolded values indicate the cut-point that maximizes sensitivity and specificity for each component

hospital setting. During and after emergencies, people are more likely to suffer from a range of mental health problems, often related to poor adjustment to hospitalization distress [45]. Some people develop new mental disorders after an emergency, while others experience temporary mental distress [11]. Our findings suggest that patients experienced more anxiety and depressive symptoms in hospital (at discharge) than in the community setting (90-days post-discharge), possibly due to the distress of hospitalization. For depression and anxiety diagnoses, anxiety and depressive symptoms need to persist for an extended period of time, usually at least 2 weeks, which is the reference period on the GAD and PHQ measures. The EQ-5D-3L reference period "today" may have impacted the prevalence of positive anxiety and/or depression screens at the time of discharge, as hospitalization is a disruptive, worrisome event that could influence patients' reporting of anxiety/depression. Further, we likely see the best screening accuracy of the EQ-5D-3L anxiety/depression dimension in the community setting because those who experienced anxiety and/ or depressive like symptoms at discharge only in relation to hospitalization, no longer have these symptoms in the community, 90-days post-discharge, and therefore do not report any problems on the EQ-5D-3L anxiety/depression dimension. Whereas, those who have anxiety and/ or depressive symptoms unrelated to the hospitalization were likely to continue to report problems and screen positive in the community setting.

Within both the hospital and community settings, the screening performance of the anxiety/depression dimension was better when anxiety and depressive symptoms were both present than either symptom alone. Previous studies have examined how composite EQ-5D dimensions, a combination of two separate but related items, such as the anxiety/depression dimension, are interpreted by respondents [46, 47]. Tsuchiya et al. (2019) found that what respondents have in mind when valuing "extreme anxiety or depression" is that both conditions are significantly worse than extreme anxiety on its own [46]. Furthermore, depression was perceived to be worse than anxiety at the same level. Our study findings are in accordance with these results given that the sensitivity is stronger for anxiety than depression and strongest when both anxiety and depression are present. However, McDonald et al. (2020) found that respondents interpret the anxiety/depression dimension as "the component of anxiety and depression with the most severe reported problems" [47]. Nonetheless, respondents seem to consider both anxiety and depression when responding to the anxiety/depression dimension which is not the case of the pain/discomfort dimension where respondents mainly use this dimension to only report pain [47].

Although mental disorders, depression in particular, have been identified as the most common diagnosis among people hospitalized with physical diseases [48], screening is not regularly done among hospitalized patients. Guidelines differ between agencies despite the availability of suitable screening tests [10] and are specified for the primary care outpatient setting. In the hospital setting, there is a pressure to provide cost and time-efficient care that discharges patients as quickly as possible [9]. It is suggested that if mental health screening were to become routine in hospital settings, screening tools need to be sensitive, specific, brief, and suitable for self-administration by patients or health care providers [9]. While, the EQ-5D-3L meets most of those criteria, the reference period "today" remains an issue for the hospitalized patient population. Mental health screening using the EQ-5D-3L in the hospital setting may be less useful in identifying long-term mental health issues and further screening in the community after discharge would be necessary.

This study has a few limitations that should be considered in interpreting its results. First, anxiety and depressive symptoms were measured by self-report. Although GAD-2 and PHQ-9 are widely used and established screening measures of anxiety and depressive symptoms, respectively, comparing the performance of the EQ-5D-3L to clinical assessments of these symptoms would enhance the robustness of these analyses. Second, the EQ-5D-3L was not developed for clinical screening or diagnosis. The reference period of the tool is "today", and each dimension asks about the severity of problems, not their frequency or impact on life. These characteristics may affect the sensitivity and specificity of the tool. For instance, disorders such as generalized anxiety tend to be highly persistent, whereas others, such as panic disorder, tend to be characterized by episodic symptoms [17]. Third, the Canadian EQ-5D-3L uses the wording 'I am not/moderately/extremely anxious or depressed. There is possible bias that respondents interpret this as a clinical diagnosis rather than a feeling, therefore, underreporting anxiety/depressive symptoms. Fourth, some of the examined groups within the overall sample had small sample sizes, also limiting the analysis. And lastly, 
Table 6 Performance of the EQ-5D-3L in screening for comorbid anxiety (GAD-2 $\geq 3$ ) with any depressive symptoms (PHQ-9 $\geq 10$ ), and comorbid anxiety (GAD-2 $\geq 3$ ) with moderate-severe depressive symptoms (PHQ-9 $\geq 15$ )

\begin{tabular}{|c|c|c|c|c|}
\hline \multirow[t]{2}{*}{ Cut-point } & \multicolumn{2}{|c|}{ HOSPITAL SETTING $(\mathrm{N}=492)$} & \multicolumn{2}{|c|}{$\begin{array}{l}\text { COMMUNITY SETTING } \\
(\mathrm{N}=400)\end{array}$} \\
\hline & $\geq 2$ & $\geq 3$ & $\geq 2$ & $\geq 3$ \\
\hline \multicolumn{5}{|c|}{$\begin{array}{l}\text { Anxiety (GAD-2 } \geq 3 \text { ) \& any depressive symptoms (PHQ- } \\
9 \geq 10 \text { ) }\end{array}$} \\
\hline \multicolumn{5}{|c|}{ EQ-5D-3L Anxiety/depression dimension } \\
\hline Sensitivity & $78.3 \%$ & $18.1 \%$ & $92.3 \%$ & $23.1 \%$ \\
\hline Specificity & $65.1 \%$ & $96.6 \%$ & $77.5 \%$ & $97.3 \%$ \\
\hline LR+ & 2.24 & 5.25 & 4.11 & 8.63 \\
\hline LR- & 0.33 & 0.85 & 0.10 & 0.79 \\
\hline PPV & $31.3 \%$ & $52.0 \%$ & $22.2 \%$ & $37.3 \%$ \\
\hline NPV & $93.7 \%$ & $85.3 \%$ & $99.3 \%$ & $94.8 \%$ \\
\hline AUROC (95\% Cl) & $0.74(0.68,0.79)$ & & $0.86(0.80,0.92)$ & \\
\hline \multicolumn{5}{|c|}{ EQ-5D-3L index score tertiles } \\
\hline Sensitivity & $48.8 \%$ & $11.9 \%$ & $15.4 \%$ & $0.0 \%$ \\
\hline Specificity & $31.9 \%$ & $65.0 \%$ & $38.0 \%$ & $70.9 \%$ \\
\hline LR+ & 0.72 & 0.34 & 0.25 & 0.00 \\
\hline LR- & 1.61 & 1.36 & 2.23 & 1.41 \\
\hline PPV & $12.7 \%$ & $6.5 \%$ & $1.7 \%$ & $0.0 \%$ \\
\hline NPV & $75.4 \%$ & $78.4 \%$ & $86.7 \%$ & $91.1 \%$ \\
\hline AUROC (95\% Cl) & $0.36(0.30,0.41)$ & & $0.24(0.19,0.30)$ & \\
\hline \multicolumn{5}{|l|}{ EQ-VAS score tertiles } \\
\hline Sensitivity & $48.2 \%$ & $9.6 \%$ & $26.9 \%$ & $7.7 \%$ \\
\hline Specificity & $31.5 \%$ & $72.2 \%$ & $32.4 \%$ & $70.1 \%$ \\
\hline LR+ & 0.70 & 0.35 & 0.40 & 0.26 \\
\hline LR- & 1.65 & 1.25 & 2.26 & 1.32 \\
\hline PPV & $12.5 \%$ & $6.6 \%$ & $2.7 \%$ & $1.8 \%$ \\
\hline NPV & $74.9 \%$ & $79.7 \%$ & $86.4 \%$ & $91.6 \%$ \\
\hline AUROC (95\% Cl) & $0.36(0.31,0.42)$ & & $0.28(0.19,0.37)$ & \\
\hline \multicolumn{5}{|c|}{$\begin{array}{l}\text { Anxiety (GAD-2 } \geq 3) \& \text { moderate-severe depressive symp- } \\
\text { toms (PHQ-9 } \geq 15)\end{array}$} \\
\hline \multicolumn{5}{|c|}{ EQ-5D-3L Anxiety/depression dimension } \\
\hline Sensitivity & $80.8 \%$ & $17.3 \%$ & $100.0 \%$ & $40.0 \%$ \\
\hline Specificity & $62.3 \%$ & $95.4 \%$ & $74.9 \%$ & $96.9 \%$ \\
\hline LR+ & 2.14 & 3.79 & 3.98 & 13.00 \\
\hline$L R-$ & 0.31 & 0.87 & 0.00 & 0.62 \\
\hline PPV & $20.3 \%$ & $30.8 \%$ & $6.8 \%$ & $19.1 \%$ \\
\hline NPV & $96.5 \%$ & $90.7 \%$ & $100.0 \%$ & $98.9 \%$ \\
\hline AUROC (95\% Cl) & $0.73(0.67,0.79)$ & & $0.91(0.87,0.95)$ & \\
\hline \multicolumn{5}{|c|}{ EQ-5D-3L index score tertiles } \\
\hline Sensitivity & $45.3 \%$ & $11.3 \%$ & $10.0 \%$ & $0.0 \%$ \\
\hline Specificity & $32.8 \%$ & $66.5 \%$ & $39.7 \%$ & $72.1 \%$ \\
\hline LR+ & 0.67 & 0.34 & 0.17 & 0.00 \\
\hline LR- & 1.67 & 1.33 & 2.26 & 1.39 \\
\hline PPV & $7.4 \%$ & $3.9 \%$ & $0.3 \%$ & $0.0 \%$ \\
\hline NPV & $83.5 \%$ & $86.3 \%$ & $96.0 \%$ & $97.5 \%$ \\
\hline AUROC (95\% Cl) & $0.35(0.29,0.42)$ & & $0.23(0.16,0.31)$ & \\
\hline \multicolumn{5}{|c|}{ EQ-VAS score tertiles } \\
\hline Sensitivity & $40.4 \%$ & $3.9 \%$ & $28.6 \%$ & $14.3 \%$ \\
\hline Specificity & $32.0 \%$ & $72.8 \%$ & $34.2 \%$ & $71.3 \%$ \\
\hline
\end{tabular}


Table 6 (continued)

\begin{tabular}{|c|c|c|c|c|}
\hline \multirow[t]{2}{*}{ Cut-point } & \multicolumn{2}{|c|}{ HOSPITAL SETTING $(\mathrm{N}=492)$} & \multicolumn{2}{|c|}{$\begin{array}{l}\text { COMMUNITY SETTING } \\
(\mathrm{N}=400)\end{array}$} \\
\hline & $\geq 2$ & $\geq 3$ & $\geq 2$ & $\geq 3$ \\
\hline LR+ & 0.59 & 0.14 & 0.43 & 0.50 \\
\hline LR- & 1.87 & 1.32 & 2.09 & 1.20 \\
\hline PPV & $6.6 \%$ & $1.7 \%$ & $0.8 \%$ & $0.9 \%$ \\
\hline NPV & $81.9 \%$ & $86.5 \%$ & $96.3 \%$ & $97.8 \%$ \\
\hline AUROC $(95 \% \mathrm{Cl})$ & \multicolumn{2}{|c|}{$0.32(0.26,0.38)$} & \multicolumn{2}{|c|}{$0.32(0.12,0.52)$} \\
\hline
\end{tabular}

$\mathrm{LR}+$, positive likelihood ratio; $\mathrm{LR}-$, negative likelihood ratio; $\mathrm{AUROC}$, area under receiver operating curve. $E \mathrm{E}-5 \mathrm{D}-3 \mathrm{~L}$ index score tertiles: $2=$ quintile $2,3=$ quintile 3. EQ-5D-3L anxiety/depression dimension: 2 =moderately anxious or depressed, $3=$ extremely anxious or depressed. PPV, positive predictive value; NPV, negative predictive value. Bolded values indicate the cut-point that maximizes sensitivity and specificity for each component

this study included predominantly Caucasian older-aged adults in Alberta, Canada, limiting the generalizability of the results to similar populations.

\section{Conclusions}

Our findings suggest that the EQ-5D-3L anxiety/depression dimension could be a useful screening tool for anxiety and depressive symptoms, compared to other self-report screening tools, in community settings. The performance of this tool in the hospital setting was poor. While screening does not replace clinical diagnosis, this tool may help in identifying patients with anxiety and/or depressive symptoms that otherwise may not be identified. Examining the screening performance of EQ-5D-3L and other patient-reported outcome measures in other populations and settings is warranted.

\section{Abbreviations \\ ANOVA: Analysis of variance; AUROC: Area under receiver operating curve; EQ VAS: EuroQol Visual Analogue Scale; GAD-2: Generalized Anxiety Disorder 2-item; PHQ-9: Patient Health Questionnaire 9-item; PROMs: Patient-reported outcome measures; QALY: Quality-adjusted life year.}

\section{Authors' contributions}

HS made substantial contributions to analysis and interpretation of data and was involved in drafting the manuscript. FAS made substantial contributions to conception of study and was involved in revising the manuscript critically for important intellectual content. AO made contributions to the conception of the study and revised the manuscript for intellectual content. JJ was involved in the conception of the study; revised the manuscript critically for important intellectual content; gave final approval of the version to be published; agreed to be accountable for all aspects of the work in ensuring that questions related to the accuracy or integrity of any part of the work are appropriately investigated and resolved. All authors read and approved the final manuscript.

\section{Funding}

This study was conducted at Alberta PROMs and EQ-5D Research and Support Unit (APERSU), which is funded by Alberta Health Services, Health Quality Council of Alberta and Alberta Health.

\section{Availability of data and materials}

The data that supports the findings of this study are available from the University of Alberta but restrictions apply to the availability of these data, which were used under license for the current study, and so are not publicly available. Data are however available from the authors upon reasonable request and with permission of the University of Alberta.

\section{Code availability}

Not applicable.

\section{Ethics approval and consent to participate}

The Research Ethics Board at the University of Alberta reviewed and approved survey questions and data collection protocols. Informed consent was obtained from all individual participants included in the study.

\section{Research involving human participants}

The study design was approved by the Health Research Ethics Board at the University of Alberta, protecting participants' human rights, privacy, and confidentiality.

\section{Consent for publication}

Not applicable.

\section{Competing interests}

Fatima Al Sayah, Arto Ohinmaa, and Jeffrey Johnson are members of the EuroQol Group. The authors declare that they have no competing interests.

\section{Author details}

1 2-040 Li Ka Shing Centre for Health Research Innovation, School of Public Health, University of Alberta, Edmonton, AB T6G 2E1, Canada. ${ }^{2}$ 3-267 Edmonton Clinic Health Academy, School of Public Health, University of Alberta, Edmonton, AB, Canada.

Received: 22 October 2020 Accepted: 4 March 2021

Published online: 19 March 2021

References

1. Rayner L, Matcham F, Hutton J, et al. Embedding integrated mental health assessment and management in general hospital settings: feasibility, acceptability and the prevalence of common mental disorder. Gen Hosp Psychiatry. 2014;36(3):318-24.

2. Mood Disorders Society of Canada. Quick facts: mental illness \& addiction in Canada. 2019

3. Mental Health Commission of Canada. Strengthening the case for investing in Canada's mental health system: economic considerations. Ottawa, ON2017.

4. Sunderland A, Findlay LC. Perceived need for mental health care in Canada: results from the 2012 Canadian Community Health SurveyMental Health. Health Rep. 2013;24(9):3-9.

5. Clarke DM, Minas IH, Stuart GW. The prevalence of psychiatric morbidity in general hospital inpatients. Aust N Z J Psychiatry. 1991;25(3):322-9.

6. Seltzer A. Prevalence, detection and referral of psychiatric morbidity in general medical patients. J R Soc Med. 1989;82(7):410-2. 
7. Moffic HS, Paykel ES. Depression in medical in-patients. Br J Psychiatry. 1975;126:346-53.

8. Gous P, Gagiano CA, Weich DJ. Psychiatric disorders in internal medicine. S Afr Med J. 1992;82(6):441-2.

9. IsHak WW, Collison K, Danovitch I, et al. Screening for depression in hospitalized medical patients. J Hosp Med. 2017;12(2):118-25.

10. Al Sayah F, Ohinmaa A, Johnson JA. Screening for anxiety and depressive symptoms in type 2 diabetes using patient-reported outcome measures: comparative performance of the EQ-5D-5L and SF-12v2. MDM Policy Pract. 2018. https://doi.org/10.1177/2381468318799361.

11. World Health Organization. Mental health in emergencies. https://www. who.int/en/news-room/fact-sheets/detail/mental-health-in-emergencie s. Accessed May 21, 2019.

12. Dennis M, Kadri A, Coffey J. Depression in older people in the general hospital: a systematic review of screening instruments. Age Ageing. 2012:41(2):148-54.

13. Albrecht JS, Gruber-Baldini AL, Hirshon JM, et al. Depressive symptoms and hospital readmission in older adults. J Am Geriatr Soc. 2014;62(3):495-9.

14. König H-H, Born A, Günther $\mathrm{O}$, et al. Validity and responsiveness of the EQ-5D in assessing and valuing health status in patients with anxiety disorders. Health Qual Life Outcomes. 2010;8(1):47.

15. Brazier J, Connell J, Papaioannou D, et al. A systematic review, psychometric analysis and qualitative assessment of generic preference-based measures of health in mental health populations and the estimation of mapping functions from widely used specific measures. Health Technol Assess. 2014;18(34):vii-viii, xiii-XxV, 1-188.

16. Sapin C, Fantino B, Nowicki M-L, Kind P. Usefulness of EQ-5D in assessing health status in primary care patients with major depressive disorder. Health Qual Life Outcomes. 2004;2:20-20.

17. Supina AL, Johnson JA, Patten SB, Williams JV, Maxwell CJ. The usefulness of the EQ-5D in differentiating among persons with major depressive episode and anxiety. Qual Life Res. 2007;16(5):749-54.

18. Vermani M, Marcus M, Katzman MA. Rates of detection of mood and anxiety disorders in primary care: a descriptive, cross-sectional study. Prim Care Companion CNS Disord. 2011. https://doi.org/10.4088/PCC.10m01 013.

19. Wittchen HU. Generalized anxiety disorder: prevalence, burden, and cost to society. Depress Anxiety. 2002;16(4):162-71.

20. Roy-Byrne PP, Wagner A. Primary care perspectives on generalized anxiety disorder. J Clin Psychiatry. 2004;65(Suppl 13):20-6.

21. Shear K, Belnap BH, Mazumdar S, Houck P, Rollman BL. Generalized anxiety disorder severity scale (GADSS): a preliminary validation study. Depress Anxiety. 2006;23(2):77-82

22. Spitzer RL, Kroenke K, Linzer M, et al. Health-related quality of life in primary care patients with mental disorders. Results from the PRIME-MD 1000 Study. JAMA. 1995;274(19):1511-7.

23. Broadhead WE, Leon AC, Weissman MM, et al. Development and validation of the SDDS-PC screen for multiple mental disorders in primary care. Arch Fam Med. 1995;4(3):211-9.

24. McQuaid JR, Stein MB, McCahill M, Laffaye C, Ramel W. Use of brief psychiatric screening measures in a primary care sample. Depress Anxiety. 2000;12(1):21-9.

25. Staab JP, Datto CJ, Weinrieb RM, Gariti P, Rynn M, Evans DL. Detection and diagnosis of psychiatric disorders in primary medical care settings. Med Clin N Am. 2001;85(3):579-96.

26. Katon WJ, Lin EH, Von Korff M, et al. Collaborative care for patients with depression and chronic illnesses. N Engl J Med. 2010;363(27):2611-20.

27. Sambamoorthi U, Walkup J, Olfson M, Crystal S. Antidepressant treatment and health services utilization among HIV-infected medicaid patients diagnosed with depression. J Gen Intern Med. 2000;15(5):311-20.

28. Katon W, Russo J, Lin EH, et al. Cost-effectiveness of a multicondition collaborative care intervention: a randomized controlled trial. Arch Gen Psychiatry. 2012;69(5):506-14
29. Le Fevre P, Devereux J, Smith S, Lawrie SM, Cornbleet M. Screening for psychiatric illness in the palliative care inpatient setting: a comparison between the Hospital Anxiety and Depression Scale and the General Health Questionnaire-12. Palliat Med. 1999;13(5):399-407.

30. Whooley MA, Avins AL, Miranda J, Browner WS. Case-finding instruments for depression. Two questions are as good as many. J Gen Intern Med. 1997;12(7):439-45.

31. Halbreich U. Teaching normal and abnormal behavior to primary care physicians. Int J Psychiatry Med. 1994;24(2):115-20.

32. Zimmerman M, Galione J. Psychiatrists' and nonpsychiatrist physicians' reported use of the DSM-IV criteria for major depressive disorder. J Clin Psychiatry. 2010;71(3):235-8.

33. Anxiety and Depression Association of America. Understand the Facts: Depression: Treatment. https://adaa.org/understanding-anxiety/depre ssion/treatment. Accessed June 12, 2019.

34. Kahlon S, Pederson J, Majumdar SR, et al. Association between frailty and 30-day outcomes after discharge from hospital. CMAJ. 2015:187(11):799-804.

35. Rabin R, Oppe M, Janssen B, Herdman M. EQ-5D-3L user guide. Basic information on how to use the EQ-5D-5L instrument. Rotterdam: EuroQol Group; 2011.

36. Devlin NJ, Krabbe PF. The development of new research methods for the valuation of EQ-5D-5L. Eur J Health Econ. 2013;14(Suppl 1):S1-3.

37. Xie F, Pullenayegum E, Gaebel K, et al. A time trade-off-derived value set of the EQ-5D-5L for Canada. Med Care. 2016;54(1):98-105.

38. Skapinakis P. The 2-item Generalized Anxiety Disorder scale had high sensitivity and specificity for detecting GAD in primary care. Evid Based Med. 2007;12(5):149.

39. Kroenke K, Spitzer RL, Williams JB, Monahan PO, Lowe B. Anxiety disorders in primary care: prevalence, impairment, comorbidity, and detection. Ann Intern Med. 2007;146(5):317-25.

40. Manea L, Gilbody S, McMillan D. Optimal cut-off score for diagnosing depression with the Patient Health Questionnaire (PHQ-9): a meta-analysis. CMAJ. 2012;184(3):E191-196.

41. Bombardier CH, Kalpakjian CZ, Graves DE, Dyer JR, Tate DG, Fann JR. Validity of the Patient Health Questionnaire-9 in assessing major depressive disorder during inpatient spinal cord injury rehabilitation. Arch Phys Med Rehabil. 2012;93(10):1838-45.

42. Swets JA. Measuring the accuracy of diagnostic systems. Science. 1988;240(4857):1285-93.

43. Stata Statistical Software: Release 14.2. StataCorp LP; 2015.

44. Janssen B, Szende A. Population norms for the EQ-5D. In: Szende A, Janssen B, Cabases J, editors. Self-reported population health: an international perspective based on EQ-5D. Dordrecht: Springer; 2014.

45. Tully PJ, Baker RA. Depression, anxiety, and cardiac morbidity outcomes after coronary artery bypass surgery: a contemporary and practical review. J Geriatr Cardiol. 2012;9(2):197-208.

46. Tsuchiya A, Bansback N, Hole AR, Mulhern B. Manipulating the 5 dimensions of the EuroQol instrument: the effects on self-reporting actual health and valuing hypothetical health states. Med Decis Making. 2019:39(4):379-92.

47. McDonald R, Mullett TL, Tsuchiya A. Understanding the composite dimensions of the EQ-5D: an experimental approach. Soc Sci Med. 2020;265:113323.

48. Meneghetti CC, Guidolin BL, Zimmermann PR, Sfoggia A. Screening for symptoms of anxiety and depression in patients admitted to a university hospital with acute coronary syndrome. Trends Psychiatry Psychother. 2017:39(1):12-8.

\section{Publisher's Note}

Springer Nature remains neutral with regard to jurisdictional claims in published maps and institutional affiliations. 